\title{
The Role of AI and Automation on the Future of Jobs and the Opportunity to Change Society
}

\author{
Manuel Au-Yong-Oliveira ${ }^{1,2(\sqrt{ })}$, Diogo Canastro ${ }^{1}$, Joana Oliveira ${ }^{1}$, \\ João Tomás ${ }^{1}$, Sofia Amorim ${ }^{1}$, and Fernando Moreira ${ }^{3,4}$ \\ ${ }^{1}$ Department of Economics, Management, Industrial Engineering and Tourism, \\ University of Aveiro, 3810-193 Aveiro, Portugal \\ ${ }^{2}$ GOVCOPP, Aveiro, Portugal \\ 3 IJP, REMIT, Universidade Portucalense, Porto, Portugal \\ fmoreira@upt.pt \\ ${ }^{4}$ IEETA, Universidade de Aveiro, Aveiro, Portugal
}

\begin{abstract}
In today's world, technology is an indispensable part of our daily life. More and more products are produced to satisfy the needs of a growing population and the Internet is creating new services every day. However, to be able to keep up with the growing demand, new technologies needed to be invented to increase the pace of production and to lower costs. Automating tasks was the solution and for many years machines did repetitive tasks, replaced people and created new and better jobs to substitute the old ones. Nowadays automation is reaching incredible levels and it is not creating enough jobs to replace the old ones. Will unemployment increase in the upcoming years or will humanity be able to adapt to a different job market? Our study focuses on the types of unemployment caused by automation and on the possible solutions that society needs to implement. The research also points out how people from different social classes face the changing job market and how all can benefit from it. A new form of governance of society may be needed, in view of previous failed forms including fascism, communism, and, more recently, liberalism. In the future there will be fewer and fewer jobs that cannot be replaced by a robot. With this, the probability of mass unemployment is very high. An ideology promoting a Universal Basic Income could be a solution to combat the massive unemployment that automation may cause in the future, especially in medium-skilled jobs.
\end{abstract}

Keywords: Automation $\cdot$ Industry $\cdot$ Unemployment $\cdot$ Artificial Intelligence

\section{Introduction}

In the last 100 years, Automation has been applied in several kinds of fields such as services, industry or personal devices. Many inventions were created to replace human labor and others to improve jobs that already existed. In factories, robots were developed to do repetitive handiwork without making human errors and increasing the

(C) Springer Nature Switzerland AG 2019

Á. Rocha et al. (Eds.): WorldCIST'19 2019, AISC 932, pp. 348-357, 2019.

https://doi.org/10.1007/978-3-030-16187-3_34 
pace of production. In our homes, we can watch a movie without the need to find one. With the simple pressing of a button, Netflix or another streaming platform will advise us as to what movie we should watch, using its powerful algorithm. And while we are shopping at the supermarket, we can scan the bar code of the products we want and pay at the exit without the need to get the products out of the shopping cart - putting them on the cash register mat, waiting for the employee to scan the bar codes and putting the products in the shopping cart again. In sum, automation benefits our life in multiple ways, making our tasks easier, faster and more convenient.

But talking about automation, we cannot forget about an important part of this subject: Artificial Intelligence (AI). We can define AI as a part of computer science that develops systems capable to perform tasks that required human intelligence and are able to adapt to different scenarios [1]. Although a big portion of people do not know how to define what artificial intelligence is, they come into contact with it every day and it is not a strange thing to them.

Automation and AI have changed our way of life and are more present than ever before in our daily tasks. Human kind is getting more and more dependent on new technologies that are able to do tasks with a minimum of interaction with people. Machines have taken over tasks that were entirely done by people. Although this is a very good improvement in our daily life that can help us get more time to do other things, from a job point of view it is becoming a big problematic discussed by numerous specialists.

Automation appeared with a single purpose: to let machines perform repetitive and monotonous tasks. This gave the opportunity for people to transit from low skill jobs in the industry to medium and high skill jobs in services and even in the same industry.

But adding Artificial Intelligence to the equation makes this subject even more complex. Machines have become so smart that they can mimic the more complex tasks that many years ago most people did not think was possible. Various specialists have different opinions on what will happen in the future. The majority think that unemployment will reach a new high and others think this will offer better jobs to everyone.

For example, according to a study done by Boston University, since the first implementation of the Automated Teller Machine (ATM), the number of human tellers has grown considerably [2]. These numbers raise an important question. Why automation in this sector has not eliminated or, at least decreased, the number of employers? And although the first ATM was installed in 1967, can we compare it with today's and future automation innovations?

In this article, the authors will explain this battle and present their own perspective.

\section{Literature Review}

As was already explained, there is a conflict between automation and general employment. If the machines can do our work, why are we still working? And will this happen the same way in the future? 
Every company needs workers with a multiplicity of skills. With only automated systems, it is not possible to achieve this goal. It emphasizes the need for human work. Since there is a dependency on the control of machines, the value of specialized people increases.

According to David Autor, we can use two economic principles to explain this fact. The first principle is the O-ring principle. It analyses human creativity and capability [3]. As an example, we can think about the implementation of the first Automated Teller Machines (ATM). It had two different consequences on employment. As we can imagine, in the beginning, there was a lot of hand work being replaced by ATMs. These machines handle cash tasks faster.

With this transformation, banks started to open new branches because of the lower costs. More branches meant more tellers but with different work. They started a new business and adapted their jobs. Instead of doing the cash-handling tasks, they bet on a relationship with customers, by choosing a more cognitively demanding job and introducing new products (for example, investments or credit cards) [2].

By analyzing the teller's reaction, it is possible to comprehend human economic value. Since machines were not able to relate to the customer, the importance of human work and the capability to adjust to market changes was clear (problem-solving and judgment skills).

This recognition of the importance of human work is present in many jobs. A professor can be substituted by online/digital classes, but it will never compensate the relationship between a student and a teacher. It will not draw the student into the passion of learning and the exchange of knowledge.

The evolution of automatization magnifies the importance of human labor by selecting the type of work that can only be done by people. When only the first principle is used, the importance of human work is easily known, but it is not possible to know how many people are needed to do the job. Here is when the second principle is needed, the Never-get-enough principle. It analyses human insatiability [3].

Most of today's work only started to exist in the last century. But why? Every day, there is a constant need to look for new ideas, new products, new technologies or even new services. People always want more and are willing to invest time and money to find or create this $[4,5]$. This greed supports innovation in companies and creates more types of careers. Not just the ones that control the machines and the automatization but also the creative and marketing jobs. They need to be the first to capture their consumer's attention with innovative products.

So, if we accept these two principles, does it mean that there is an employment problem? No. We need to take into consideration the evolution of automatization and innovation.

Alex Williams, in his article called "Will Robots Take Our Children's Jobs?" wonders if Artificial Intelligence could make countless professions obsolete in a very near future [6]. Professions like radiologists, lawyers and surgeons are being threatened by intelligent machines. Arterys, a start-up company, has created software that can perform a magnetic-resonance imaging analysis of blood flowing through a heart in $15 \mathrm{~s}$ while a human needs $45 \mathrm{~min}$ to perform this [7]. And are professions like journalism safe in The Information Age? According to another article the answer is no. The Associated Press already used a program to produce a copy covering Wall Street 
earnings and some college sports [7]. A study done in 2013 by the Department of Science Engineering of the University of Oxford estimated that $47 \%$ of current jobs will be victims of automation, in a period of 20 years [8]. By 2033, new jobs certainly will have been created, but will they be enough to compensate for the jobs that were eliminated?

Because of this changing environment, there is also the problem that society does not know what are the skills that we should teach to children because the skills that are needed today, are not the same as those that will be needed in 20 or 30 years from now [9-11].

\section{Methodology}

Initially, a work study was conducted in order to analyze the current situation in the job market caused by automation and to identify the main causes of unemployment.

To arrive at conclusions about automation in the industry field, a questionnaire was created, having as a target audience the lower working-class in industry. The authors aimed to study the knowledge of the workers about the famous concept "Industry 4.0", that is, if they were aware of what tasks automation could do and the impact that it could have on employability in the upcoming years.

For this to be able to happen, companies were contacted, and the authors asked their permission to distribute the questionnaires to their employees. Six companies in the center/north region of Portugal were contacted, which asked to remain anonymous, but didn't give the permission needed. One of the persons who provided an explanation referred that by asking those questions to the operators it could have a negative effect on their perception of new automated technologies that the company would implement in the future and cause some discomfort in the work environment.

With this, it was realized that asking this type of questions could easily have a big impact on the satisfaction of factory workers and that those people might suspect of future investments of their company in automated jobs.

Since it was not possible to make the questionnaire, the authors chose to analyze more scientific articles and more case studies with real examples from different companies.

The authors started to analyze which jobs are more at risk of being substituted by machines. After this, it was necessary to investigate what people think about new technologies in their work space and how they adapt to a changing environment. Various future possible scenarios were already thought of by numerous experts and the ones analyzed were those that the authors think are the most probable. A possible solution was also discussed.

\section{A Theoretical Model}

There are three types of employment. The first is high-wage jobs with high-education. It represents work like that of engineers, sales and marketing managers, programmers or doctors. The second is middle-class jobs like operative positions. The low-skill is the 
last type and it represents low-education jobs (for example, cleaning service or home health aides).

According to David Autor, at TEDex Cambridge in 2017, the high-education and high-wage jobs are very likely to not be replaced by machines and even increase the number of jobs available. Regarding medium-skill jobs, the number available is decreasing and making people with not so much education getting jobs that don't require as much skills as they have. Those middle-skills jobs are getting substituted by machines and algorithms that understand the procedures needed to get the job done [3].

The best jobs are becoming more and more specialized. The first robots were created to substitute repetitive operations and predictable tasks. With the evolution of automation, this is no longer true. In 2017, Google's AlphaGo Software defeated a nineteen-year-old Chinese master at Go, considered the most complicated board game in the world that not even the best players can explain what they are doing [10]. This means that machines are capable of doing more types of jobs, and people need to invest more in their education and specialization if not to be replaced my machines.

According to Frey and Osborne [8], that distinguish jobs between high, medium and low risk occupations, came up with the following graphic to explain what is going to be probability of computerization, regarding the kind of jobs that were mentioned below.

In sum, Frey and Osborne [8] show that the high skill jobs like Management, Business, Finance, Computers, Engineering, Science and Services are amongst the sectors with lower probability of computerization. On the other hand, for the low skill jobs like Transportation, Material Moving, Production, Sales, Office and Administrative Support the probability of computerization is higher, since they are related to simple, monotonous and easy tasks that can easily be replaced by automation.

And what about Portugal? According to an article from Bruegel, and cited by the World Economic Forum, around 60\% of jobs are at risk of suffering computerization or automatization. This is a high percentage compared to less than $47,5 \%$ in the UK and Sweden [13].

To try to solve this problem that will affect all countries in the world even if they are underdeveloped, the authors found various articles that talk about what are the opinions of people about the implementation of new automated machines in their work space.

According to Melonee Wise, CEO of Fetch Robotics, when a company presents a new machine or new software to their employees, there are usually 5 stages of acceptance [14]:

1. The first stage is Fear. This happens because people think that those robots are there to substitute them, but most of the time they are there to make their jobs better.

2. The second stage is apprehension. The employees see that the robots will not replace them but think that they will not be qualified to interact with them.

3. The third stage is curiosity. After the employees start to work with them, they wonder what tasks the robots can do.

4. The fourth stage is tolerance. In this stage, the productivity increases because employees are more used to the new machine. 
5. And last stage is satisfaction. Usually workers like the new job experience and it facilitates their job.

This might be true in this situation, but in other situations the robots are there to substitute workers and not to help them. Look at the example of Amazon Go. Amazon launched three new supermarkets without any employees that works completely autonomously and eliminates the need for hiring workers [15]. In this case automation might be good for employers and clients but it is not good for workers.

According to Viktor Weber, Founder \& Director of the Future Real Estate Institute, there are 4 possible scenarios for this problematic [12]:

1. Automation might automate only some tasks and in other cases human labor would do the jobs. This could happen if human labor is cheaper than investing in new machines.

2. Automation would still evolve, but with limitations. Society would limit the autonomous machines in areas were human labor is an important part and it is more valuable.

3. Humans would focus on an "experience-based economy" where tasks like cooking or handicraft are more valued in society instead of engineers or economists.

4. Automation will not have limitations and will automate every task possible. Only one small part of people would profit with this and a big part of society would be unemployed. Social inequality would increase dramatically.

\section{Discussion}

With the First Industrial Revolution (1820-1840), human work got easier and productivity rose. Services and products could be produced in a shorter time while using the same number of workers. While this innovation eliminated a lot of jobs, at the same time a lot were created. It should be mentioned that those jobs were better than the previous ones due to their complexity. Workers put down manual agricultural tools to start handling big machines that could do the exact same job much faster and in a lighter way.

In sum, innovation led to higher productivity, fewer old jobs and many new and often better jobs. Overall, this worked well for most people and living standards improved. Meanwhile, humans shifted into service jobs and only a few decades ago in human history, the Information Age happened. Suddenly, the World changed its rules and our jobs are now taken over by machines much faster than they were in the past.

\subsection{Innovation}

Nowadays, there are new information industries appearing and growing. But, comparing to the past, they are creating less new jobs. To comprehend this problem, we analysed the car industry. One hundred years ago, cars transformed the way of life and everything around us. Everybody could find a job either directly or indirectly to this industry. Over time, there was a lot of very considerable investment until it became 
largely complete and nowadays, cars don't create as many jobs as was supposed, even if looking at electric cars that are a recent innovation. By comparison, in 2004, Blockbuster made $\$ 6$ billion US Dollars a year and had 84.000 workers, while Netflix with 4.500 employees made $\$ 9$ billion US Dollars in 2017 [16].

\subsection{New Machines and New Jobs}

Over thousands of years, human jobs became more and more specialized. While even the smartest machines are bad at doing complicated jobs, they are extremely good at doing defined and predictable tasks. That's the reason why so many jobs are disappearing.

Machines are becoming so good at breaking down complex jobs into simple ones, that for a lot of people, there will be no further room to specialize.

Machines teach themselves because we make this possible by giving a computer a lot of data about something people want to become better at. As an example: if a person shows to a machine all the things she bought online, it will slowly learn what to recommend to her, so she buys more things. What humans created by accident, is a huge library that machines can use to learn how humans do things and learn to do them better, by giving them information and records about everything that happens. Because of this, digital machines might be the biggest problem of all and destroy the most jobs.

It is not enough to substitute old jobs with new ones. There is a need to generate new jobs constantly because the world population is growing, and in the past, mankind has solved this through innovation. People were sure that with rising productivity, more and better jobs would be created.

This time, the nature of innovation in the Information Age is different from everything that was encountered before. Self-driving cars have been created. Selfworking robots have been created. Speaking robots have been created. And so on. It seems like innovation is going too far.

\subsection{A Solution that Can Change Society}

In the future there will be fewer and fewer jobs that cannot be replaced by a robot. With this, the probability of mass unemployment is very high. And what can one do about it? It is a massive social challenge that humankind is not prepared to face.

Various experts have already suggested a solution that we consider that is a great solution for the massive unemployment that automation may cause in the future. That solution is called Universal Basic Income (UBI).

UBI is considered the most ambitious social policy of our times. According to the Basic Income European Network (BIEN), UBI is a way to improve our social structure. Each person would receive an amount of money periodically without the need of work or of any other form of payment. That amount of money would be enough to cover all basic expenses needed for the person to survive above the poverty line [17].

This brings many questions and there are many skeptics about it that say that people would stop working and spend that amount of money on unnecessary items. Tests run in Canada in the 1970s showed that around 1\% of the recipients stopped working, mostly to take care of their kids. On average, people reduced their working hours by 
less than $10 \%$ and the extra time was used to achieve goals like going back to school or looking to get better jobs [18].

The easiest way to pay for a UBI is to end all welfare and use the free funds to finance it. Although this can be a good solution, it could also leave many people worse off than before. The second way to make this possible is higher taxes especially for the very wealthy. Nowadays the gap between the rich and the poor is rapidly increasing and UBI would maybe solve this problem [19].

UBI would be a solution to combat the massive unemployment that automation may cause in the future, especially in medium-skilled jobs. However, if everyone receives a basic income, would people still want to have low-skilled jobs that are often bad and unpopular? Some say that UBI might give them enough leverage to demand better pay and working conditions. There would still be poor and rich people, but the conditions of living of the poor would be substantively better.

So, is UBI a good idea? The honest answer is that no one knows. There needs to be a lot more research and experiments to know the right answer. Society needs to think about what kind of UBI it wants and what it is prepared to give up to pay for it. The potential is huge. It might be the most optimistic model to sustainably eliminate poverty. It might seriously help humanity to be much less stressed out.

\section{Conclusions and Suggestions for Future Research}

In a world where digital technologies are so present in people's daily lives, it is difficult to imagine a world without them, although not many years ago, most people lived in rural areas and their livelihood was from agriculture and handicrafts. In the last 100 years, the world has changed dramatically, and the pace of innovation will increase even more in the upcoming years.

Most people when they think about technology think about the products and services that they interact with in their daily lives. Most people do not worry about the technologies that are being implemented in factories and services that automate completely tasks that before were done by people.

In our view, we perceive that automation will create a high percentage of unemployment that will affect mainly people with an education. People with a high- and lowlevel education will not be affected as much as people with a medium-level education. Low skill jobs that do not require any specialization and usually are the worst paid jobs will increase and the differences between them and high-skill jobs will also increase causing a massive inequality between those two social classes. This will happen because those jobs are cheap and implementing machines or automating their tasks will not be economically worth it.

In our point of view, there is no doubt that automation will change the job market even more than it has already changed it. Although this will implicate serious problems, society should not give up on this. Automation is a good thing. Humankind just needs to find a way to adapt.

The Information Age could be a huge opportunity to solve poverty and the biggest problems facing society. Mankind just needs to think in a faster and different way. And that is one of the problems of Automation: although there is already a notion about 
what automation can do as regards unemployment, there is no major concern about it. People think this is a subject that will happen in many years from now, but they are wrong. This future is already here.

In our point of view, UBI would be a solution that could help solve many problems and not only the mass unemployment caused by automation. Tests are already ongoing in Finland and many developed countries, like Iceland, are already discussing it. However, that is not enough. There should be more tests and analysis about this solution and in an even faster manner. Although the mass unemployment caused by automation and the solution that is UBI are already discussed throughout the world, they are not discussed at the pace that they should be to accompany the transformation in industry and services.

A new form of governance of society may be needed, in view of previous failed forms including fascism (which did not endure the II World War), communism (which failed in the 1980s), and, more recently, liberalism (which has shown signs of failing, with Brexit and the rise of Donald Trump, in the USA) [20]. Globalization and liberalism have benefited only small elites, leaving the masses at the mercy of new waves of technology, which threaten their professions and livelihood [20]. An ideology promoting a UBI could be a solution. Capitalism thus allied to a form of widespread social responsibility may be a way forward for mankind.

Will Automation continue to benefit society and will UBI be the right solution to solve the problems caused by it? No one knows. But we hope that with our discussion we have provided information necessary for future research and showed that solutions need to be found faster.

\section{References}

1. Artificial intelligence | Definition of Artificial Intelligence in English by Oxford Dictionaries. https://en.oxforddictionaries.com/definition/artificial_intelligence. Accessed 27 Oct 2018

2. Bessen, J.E.: How computer automation affects occupations: technology, jobs, and skills. Boston University School of Law, Law \& Economics Working Paper No. 15-49 (2016)

3. Autor, D.: Why are there still so many jobs? The history and future of workplace automation. J. Econ. Perspect. 29(3), 3-30 (2015)

4. Martins, J., Gonçalves, R., Pereira, J., Cota, M.: Iberia 2.0: a way to leverage Web 2.0 in organizations. In: 2012 7th Iberian Conference on Information Systems and Technologies (CISTI), pp. 1-7. IEEE (2012)

5. Branco, F., Gonçalves, R., Martins, J., Cota, M.: Decision support system for the agri-food sector-the Sousacamp group case. In: New Contributions in Information Systems and Technologies, pp. 553-563. Springer, Cham (2015)

6. The New York Times: Will Robots Take Our Children's Jobs? https://www.nytimes.com/ 2017/12/11/style/robots-jobs-children.html. Accessed 21 Oct 2018

7. Arterys Company Profile, MedTech Innovator. https://medtechinnovator.org/company/ arterys/. Accessed 06 Nov 2018

8. Frey, C.B., Osborne, M.A.: The future of employment: how susceptible are jobs to computerisation? Oxford Martin Programme on Technology and Employment, Oxford Martin School, University of Oxford Working Paper, September 2013 
9. Harari, Y.N.: The Rise of the Useless Class (2017). https://ideas.ted.com/the-rise-of-theuseless-class/. Accessed 16 Oct 2018

10. Au-Yong-Oliveira, M., Gonçalves, R., Martins, J., Branco, F.: The social impact of technology on millennials and consequences for higher education and leadership. Telematics Inform. 35, 954-963 (2018)

11. Martins, J., Branco, F., Gonçalves, R., Au-Yong-Oliveira, M., Oliveira, T., Naranjo-Zolotov, M., Cruz-Jesus, F.: Assessing the success behind the use of education management information systems in higher education. Telematics and Inform. (2018, in press)

12. The New York Times: Google's AlphaGo Defeats Chinese Go Master in Win for A.I. (2017). https://www.nytimes.com/2017/05/23/business/google-deepmind-alphago-gochampion-defeat.html. Accessed 19 Oct 2018

13. Mesnard, X.: What happens when robots take our jobs? World Economic Forum (2016). https://www.weforum.org/agenda/2016/01/what-happens-when-robots-take-our-jobs/. Accessed 21 Oct 2018

14. Wise, M.: The 5 Stages of Acceptance as Robots Enter the Workforce, World Economic Forum (2018). https://www.weforum.org/agenda/2018/10/robots-are-coming-to-yourworkplace-here-s-how-to-get-along-with-them/. Accessed 19 Oct 2018

15. Wingfield, N., Mozur, P., Corkery, M.: Retailers Race Against Amazon to Automate Stores, The New York Times (2018)

16. Feldman, D.: Netflix has Record-Breaking Fourth Quarter in 2017, Exceeds \$11B in Revenue, Forbes (2018)

17. Basic Income Earth Network: What is Basic Income? https://basicincome.org/basic-income/. Accessed 23 Oct 2018

18. Flowers, A.: What Would Happen if We Just Gave People Money? FiveThirtyEight (2016). https://fivethirtyeight.com/features/universal-basic-income/. Accessed 20 Oct 2018

19. Straubhaar, T.: On the economics of a universal basic income. Interecon. Rev. Eur. Econ. Policy 52(2), 74-80 (2017)

20. Harari, Y.N.: 21 Lessons for the $21^{\text {st }}$ Century. Jonathan Cape, London (2018) 